\title{
Ebola Impact and Quarantine in a Network Model
}

\author{
Anca Rădulescu*, Joanna Herron \\ Department of Mathematics, State University of New York at New Paltz, New Paltz, NY \\ Student: herronj1@hawkmail.newpaltz.edu \\ Mentor:radulesa@nerwpaltz.edu
}

\begin{abstract}
Much effort has been directed towards using mathematical models to understand and predict contagious disease, in particular Ebola outbreaks. Classical SIR (susceptible-infected-recovered) compartmental models capture well the dynamics of the outbreak in certain communities, and accurately describe the differences between them based on a variety of parameters. However, repeated resurgence of Ebola contagions suggests that there are components of the global disease dynamics that we don't yet fully understand and can't effectively control.

In order to understand the dynamics of a more widespread contagion, we placed SIR models within the framework of dynamic networks, with the communities at risk of contracting the virus acting as nonlinear systems, coupled based on a connectivity graph. We study how the effects of the disease (measured as the outbreak impact and duration) change with respect to local parameters, but also with changes in both short-range and long-range connectivity patterns in the graph. We discuss the implications of optimizing both these measures in increasingly realistic models of coupled communities.
\end{abstract}

\section{KEYWORDS}

Epidemic Spread; Network Dynamics; Network Connectivity; Coupled Differential Equations; Compartmental Model; Information Transfer; Outbreak Impact; Outbreak Duration

\section{INTRODUCTION}

Background to Ebola and modeling

The evolution, prognosis and spreading of contagious diseases has been studied for a long time, with a variety of approaches. ${ }^{5,3,23}$ While huge progress has been made in efficiently applying containment methods ${ }^{22,38}$ and treatment ${ }^{50}$ in most cases, Ebola remains the 21 st century's taunting example of a disease which mankind does not seem prepared to handle, even at small scales. ${ }^{12,18}$

While the course of the illness is quite drastic and fatality rates have ranged in past outbreaks from $45-90 \%{ }^{46}$ (even with prompt clinical intervention), it has been argued that Ebola, with a relatively small reproduction number $1 \leq R_{0} \leq 7$ (varying among reports of different outbreaks), ${ }^{12,32}$ poses a lesser contagion threat than faster spreading diseases such as small pox $\left(R_{0}>7\right)$. However, the explosive Ebola contagions that resurge periodically, and in particular the recent simultaneous developments in a few countries around the world ${ }^{31}$ - seem to suggest that there are components of the disease dynamics that we don't yet fully grasp, ${ }^{16}$ as well as social, ${ }^{41}$ cultural and economic ${ }^{24}$ key parameters that we cannot yet fully control.

Much effort has been recently directed towards understanding and predicting Ebola outbreaks with the help of

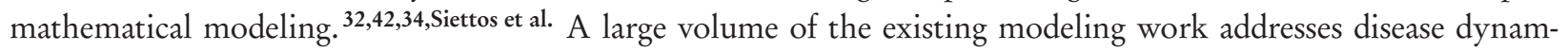
ics via low dimensional compartmental models, ${ }^{45,25,44}$ deterministic or stochastic. ${ }^{33}$ These models describe well 
outbreak dynamics in specific communities, and often explain the differences between outbreaks that occurred under different parameter regimes (e.g., geographical location, promptitude of clinical measures, timely removal of infected individuals from the community ${ }^{4,12,44}$ ). The most traditional and well-known modeling approaches to disease spread remain the SIR-type models, which describe the progress of the contagion in a single community through transfers between three main compartments: the susceptible (S), infected (I) and recovered (R) individuals. Variations of the SIR model have been vastly explored, in conjunction with parameters estimated empirically, and in increasingly complex contexts, including factors like hospitalization, treatment plans, quarantine. However, these analyses - based on localized factors and data from remote rural areas - may all be irrelevant in the context of utmost concern, that of a global outbreak, ${ }^{20,9}$ affecting urban areas as well as small communities, acting at multiple simultaneous foci. In order to understand the dynamics of a global contagion, predict its potential effects and most efficiently alter its course - one rather needs to place the problem in the mathematical framework of complex systems. ${ }^{37,36}$

The huge oriented graph that represents physical connectivity between individuals is highly modular, has hubs, multiple-scale communities (e.g., from families to towns to states), and is constantly changing due to a variety of factors (e.g. personal, economic, commuting patterns, long distance travel). To capture the global dynamics of epidemics in such a system, one must consider simultaneously the state of each node as well as its interconnections with other nodes. ${ }^{17}$ An accurate approach should bridge looking at single node dynamics to studying the effects of connectivity patterns on the systemic behavior ${ }^{6}$ and on the outbreak aftermath (asymptotic behavior). Altering dynamics in an optimal way requires knowledge of architecture, ${ }^{7}$ of the location and density of hubs and of rich clubs, ${ }^{52}$ of the robust versus vulnerable network points ${ }^{10}$ (where local edge changes produce large effects).

Networked dynamic systems ${ }^{53}$ have been used in a variety of fields with a focus on understanding the behavior of an ensemble of coupled dynamic nodes, be they cells, web servers, people, or nodes in an electric grid. However, in recent years, there has been an emerging interest in network modeling of epidemics, ${ }^{17,6,26,40}$ trying to quantify and better understand the effect of dynamic human interactions on the spread of disease between, as much as within communities, and looking to identify the factors (both deterministic and stochastic ${ }^{28}$ ) most determinant of the long term outcome.

It was noted, when studying the effects of contact heterogeneity on the dynamics of communicable disease, ${ }^{49}$ that small differences in the contact networks (e.g., taking into account restructuring at a time resolution of minutes), are typically not essential when attempting to describe disease spread on a longer timescale (of several weeks, or months). On the other hand, the same studies ${ }^{49,48}$ emphasize the importance of including detailed information about the heterogeneity of contact duration, the rate of new contacts being identified, etc. Relatively new work, including our own, is using a combination of dynamical systems and graph theoretical methods to understand how relatively small, local perturbations in connectivity patterns may produce, if targeted at the right vulnerability points, very large effects on the state of the entire network. The avenue of modeling mathematically the dynamic epidemic interactions in an infected network has been pursued in a variety of contexts, including overlay ${ }^{35}$ and adaptable networks. ${ }^{21}$ Along these lines, we have developed methods and measures of how likely the system is to undergo a sharp transition when perturbing local connectivity. ${ }^{43}$

The emergence of a contagion (of Ebola, or of any other viral or bacterial infection) can be then viewed as the propagation of a perturbation in a complex network of coupled nodes. ${ }^{29}$ One can then study how adapting the structure of the network may contribute to minimizing the global effects of the contagion. Experience has shown that, along with other important factors, timely placement of temporary travel restrictions or quarantines may be critical in isolating and extinguishing the contagion before it reaches catastrophic levels. ${ }^{19}$ One can then search for the locations where a small change in connectivity is likely to produce the largest overall effects. ${ }^{15}$ This goes along the lines of other new approaches in the field, which introduce the network structure as a system parameter, ${ }^{13,27}$ 
and search for optimal quarantine measures to efficiently isolate the epidemic.

In this paper, we consider, in conjunction with our model, two measures of outbreak effects on population: the impact, defined as the total fraction of individuals affected by the outbreak (eventually either recovered or dead), and the outbreak duration, defined as the time from the start of the outbreak (presence of the first Ebola case), until the outbreak extinguishes (less than one infected case in each community). Starting with a basic SIR model in a single, relatively small (1000 individuals) community, we construct incrementally a plausible model for Ebola spread within a network of such communities. We focus in particular on studying how our two measures of outbreak dynamics depend on the density of potential connections between these communities and on the stochastic likelihood of people to travel along these connections. The network architecture is allowed to change along the process, controlled by factors related to the current state of the outbreak, accounting for travel interdictions and quarantines typically introduced in such circumstances. Our model aims to inform on the most efficient quarantining strategies and optimal timing that would minimize life loss as well as outbreak duration.

\section{MODELING METHODS AND RESULTS}

Basic model

We build upon a basic compartmental model of Ebola due to Astacio et al., ${ }^{4}$ which was originally assembled as a classical three-variable SIR (Susceptible-Infectious-Recovery) model. In the later iterations of the model construction, the authors introduced incubation in the model, by means of a new (fourth) variable $E$, representing the "latent" (exposed) population (during the virus incubation, before development of symptoms).

$$
\begin{aligned}
\frac{d S}{d t} & =-\beta S(I+q E) / N \\
\frac{d E}{d t} & =\beta S(I+q E) / N-\delta I \\
\frac{d I}{d t} & =\delta I-\gamma I \\
\frac{d R}{d t} & =\gamma I
\end{aligned}
$$

Equation 1.

where $S(t)$ is the susceptible population at time $t$ (i.e., everyone who had not yet contracted the disease); $E(t)$ is the latent population (individuals who have contracted the virus, but are still asymptomatic); $I(t)$ represents the infected population (showing the signs and symptoms of Ebola); $R(t)$ is the number of dead or recovered individuals (i.e., in an oversimplified view, individuals who can no longer infect others with the Ebola virus). The infection rate (i.e., the rate $d E / d t=-d S / d t=\beta S(I+q E) / N$ at which people become infected) is proportional with the product of the number of susceptible individuals and the number of individuals carrying the virus (both latent and infected, with the latent individuals having a lesser impact, represented by the smaller weight $q<1$ ). The proportionality constant $\beta$ is the product of the per capita contact rate and the probability of infection after contact with an infected individual. The infection rate was normalized by the factor $N(t)$, representing the total population at time $t: N(t)=S(t)+E(t)+I(t)+R(t)$. The rate of transfer from the latent to the infectious stage is a fraction $\delta$ of the number of latent individuals - where $1 / \delta$ is the average time for a latent individual to become infectious. The rate of death/recovery is a fraction $\gamma$ of the infectious population, where $1 / \gamma$ is the average time it takes a person to die or recover once in the infectious stage.

The parameters were based on the disease dynamics described in a relatively well localized 1976 contagion in Yambuku, Zaire. The ranges of values, as per our reference, are listed in Table 1. In our study, we worked primarily with these values; changes and extensions, based on information on post-mortem and post-recovery potential for 
contagion, are explored in the Discussion. In this study, however, we work under the same simplifying assumption as the one made by Astacio et al.: ${ }^{4}$ that neither deceased nor recovered individuals can contribute any further to the perpetuation of the disease cycle, hence are represented by a common variable, whose asymptotic value $R(t)$, whose asymptotic value as $t$ approaches $\infty$ can be viewed as measuring the impact of the outbreak (total fraction of people affected).

\begin{tabular}{|c|c|c|c|}
\hline Parameter & Range & Value & Units \\
\hline $1 / \gamma$ & $4-10$ & 7 & days \\
\hline $1 / \delta$ & $2-21$ & 12 & days \\
\hline$q$ & - & 0.25 & - \\
\hline$\beta$ & - & 0.567 & - \\
\hline
\end{tabular}

Table 1. Parameter values for the Yumbuku outbreak, as per the Astacio et al. reference. ${ }^{4}$

Simple network model

We first studied contagion propagation in a small, unstructured network of interconnected communities. Although this preliminary model makes a few coarse and rather unrealistic simplifying assumptions, this first stage helps understand some very basic problems and questions to address.

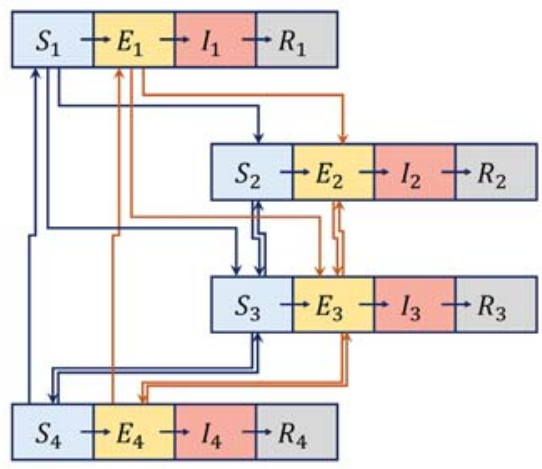

A.

Figure 1. Model network, for $n=4$ communities, (as used in some of our later simulations). A. Illustration of the network, showing each population as a compartmental node, with its 4 coupled SEIR variables, and showing transfer between populations as oriented arrows from the original population to the target population. B. Schematic representation of the same network, in which each population is viewed as a node, and the connections between populations are viewed as oriented edges. C. Adjacency matrix $C$ corresponding to the connectivity graph described in (B).

We considered a graph in which the $n$ nodes represent the interconnected communities, and the oriented edges connecting node pairs represent one-way communication between the two respective nodes. Each node/population $P_{k}, 1 \leq k \leq n$, represents a standard SEIR unit (as described in the previous section), characterized by a 4dimensional variable $\left(S_{k}, E_{k}, I_{k}, R_{k}\right)$. The communication between nodes was set up, for consistency, also in a compartmental fashion, so that a fraction of individuals travels through each existing outgoing edge from a specific node to the corresponding adjacent nodes. For simplicity, each node was assumed to have originally $N_{0}=1000$ individuals. The total number of individuals $N(t)=\sum\left[S_{k}(t)+E_{k}(t)+I_{k}(t)+R_{k}(t)\right]$ is constant throughout the 
outbreak (that is, external effects such as travel in and out of the network, birth rate and death rate due to factors independent of the disease were ignored). The corresponding coupled dynamics can then be extended from the system Equation 1. to the following system:

$$
\begin{aligned}
\frac{d S_{k}}{d t} & =-\beta S_{k}\left(I_{k}+q E_{k}\right) / N_{0}+q_{c}[C S]_{k}-q_{c} \sum_{j} C_{j, k} S_{k} \\
\frac{d E_{k}}{d t} & =\beta S_{k}\left(I_{k}+q E_{k}\right) / N_{0}+q_{c}[C E]_{k}-q_{c} \sum_{j} C_{j, k} E_{k}-\delta E_{k} \\
\frac{d I_{k}}{d t} & =\delta E_{k}-\gamma I_{k} \\
\frac{d R_{k}}{d t} & =\gamma I_{k}
\end{aligned}
$$

Equation 2.

where $[C S]_{k}$ and $[C E]_{k}$ represent the $k$ th component of the vectors $C S$ and $C E$, respectively. The $4 n$ dimensional system Equation 2. reflects the inner SEIR dynamics of each particular node $P_{k}=\left(S_{k}, E_{k}, I_{k}, R_{k}\right)$, for $1 \leq k \leq n$, but also captures, in a compartmental way, the population flow between the nodes. The adjacency matrix $C$ is defined so that $C_{j, k}=1$ if there is directed travel (an oriented edge) from the node $P_{k}$ into the node $P_{j}$. It is zero diagonal (the graph has no self loops, since travel within one's own community is not relevant).

The number of healthy and, respectively, latent individuals leaving node $P_{k}$ at time $t$, headed towards each of the adjacent nodes $P_{j}$ is $q_{c} C_{j, k} S_{k}$ and, respectively, $q_{c} C_{j, k} E_{k}$. If there is an edge between nodes $P_{k}$ and $P_{j}$, these numbers are proportional with the existing healthy and, respectively, latent population in node $P_{k}$. For simplicity, the proportion of travelers $q_{c}$ between adjacent nodes was taken to be the same for all connected node pairs (and was fixed to $q_{c}=2.5 \%$ in our simulations of this system). The rate out of $S_{k}$ due to travel to all efferent nodes is therefore $q_{c} \sum_{j} C_{j, k} S_{k}$, and the rate out of $E_{k}$ due to travel is $q_{c} \sum_{j} C_{j, k} E_{k}$.

Subsequently, each node will receive an incoming flow of travelers from the nodes connected to it. More specifically, the rate due to travel of healthy people from every node $P_{j}$ into node $P_{k}$ is $q c C_{k j} S_{j}$, so that the total rate into $S_{k}$ due to travel from other nodes is $q_{c} \sum_{j} C_{k, j} S_{j}=q_{c}[C S]_{k}$. Similarly, the total rate into $E_{k}$ due to travel from other nodes is $q_{c} \sum_{j} C_{k, j} E_{j}=q_{c}[C E]_{k}$. We based this scheme on the assumption that latent individuals are able to travel out of their own community: being asymptomatic, they have not yet been diagnosed, although they pose a risk in spreading the disease, by adding themselves to the existing latent population at a different node.

Infected individuals cannot travel in our model. In addition, circulation of recovered individuals was ignored, since it would have no effect on the disease dynamics (under the assumption that they can no longer contract or spread the disease, they would only permute between the $R_{k}$ compartments of different nodes).

The model was conceived having in mind typical rural structures, where people travel along established routes between specific locations (for daily or other periodic needs), with the return not necessarily occurring immediately or along the same path (hence the oriented edges). The graph adjacency matrix $C$ delivers a complete description of the communication patterns within the network, and induces instantaneous spread of contagion. Throughout our analysis, we kept all parameters fixed and we focused primarily on how the connection density and patterns affect viral diffusion through the network.

As a start, we studied the effect of edge density on the disease dynamics, in particular on the outbreak impact and 

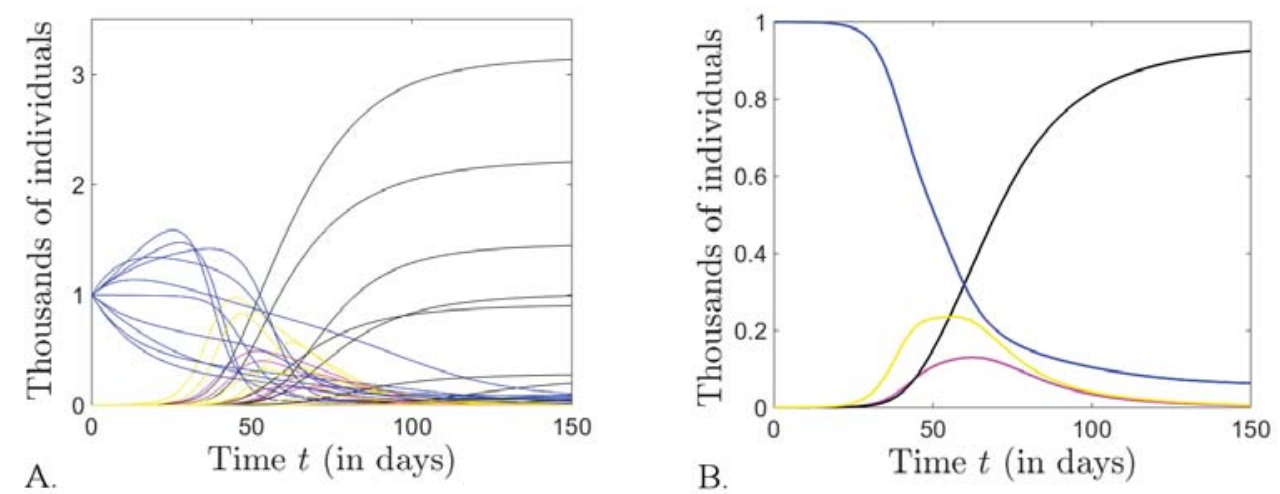

Figure 2. Simulation of interactive dynamics in a network with $n=10$ communities, in which the contagion was triggered by only one latent individual in $P_{1}$. A. The time evolution of all SEIR variables, obtained by numerically solving system Equation 2., is illustrated simultaneously for all $n=10$ nodes, with the $S_{k}$ shown in pink, the $E_{k}$ shown in yellow, the $I_{k}$ shown in red and the $R_{k}$ shown in black. B. The average populations $\bar{S}(t)=\frac{1}{n} \sum_{k=1}^{n} S(t), \bar{E}(t), \bar{I}(t), \bar{R}(t)$ are shown, with the same color coding.

duration. For our network model, we define the impact as:

$$
R_{\infty}=\lim _{t \rightarrow \infty} \bar{R}(t), \text { where } \bar{R}(t) \text { is the recovered average per community: } \bar{R}(t)=\frac{1}{n} \sum_{k=1}^{n} R_{k}(t)
$$

For this first model, we assume a constant connectivity matrix throughout the process, and we investigate the cases of a single focal point (triggered by two infections), and of two focal points (with one infection each). Fixing the network size, we ran numerical simulations for different connectivity patterns, with the primary aim of investigating the effect of the connectivity density on the outbreak coupled dynamics. For small network sizes $(n \leq 4)$, we considered all possible matrix configurations for each fixed edge density $0 \leq \Delta \leq n(n-1)$, and we computed the mean impact over all such configurations (see Figures 3 ). For larger $n$ 's, we computed a sample-based mean, over a subset of 50 configurations for each edge density $\Delta$ (Figure $4 \mathrm{a}$ and $\mathrm{b}$ ). This sample approach was preferred for larger networks, since the number of configurations increases extremely fast with $n$, making a numerical inspection of all configurations for each fixed density very expensive and impractical. In both cases, we plotted the outbreak impact and duration as functions of the edge density $\Delta$, showing both mean value and error bars (over the distribution of adjacency configurations).

Our computations suggest that, for both one and two foci, the outbreak impact increases with the density of connections. This is because the outbreak impact depends, as one might expect, on the average number of nodes connected to the foci, which in turn depends on the graph's edge density (as shown in Figure 4c). For both one and two foci, the duration has a unimodal shape (with a peak in the intermediate connectivity range). This is not a surprise either, and can be explained by the fact that for very low connectivity the contagion is quickly contained, with minimal casualties, and for very high connectivity - it produces a fast diffusion, with a high number of casualties. It is for intermediate connectivity patterns that the dynamics takes longer to develop.

Clearly, one desires to reduce duration, as well as impact (since longer infection times can be as well detrimental for the community). The two measures don't assume their minima in the same conditions. Given that most parameters of the system (such as $R_{0}$ value, number of individuals, connectivity graph) are hard-wired into the network, one may consider imposing conditions on travel along the existing edges (quarantines), in an attempt to optimize a function of both impact and duration, and assess possible regulations that would render the outcome least destructive. For example, some studies have documented the dramatic impact of long outbreaks on economy. ${ }^{9,1}$ However, 

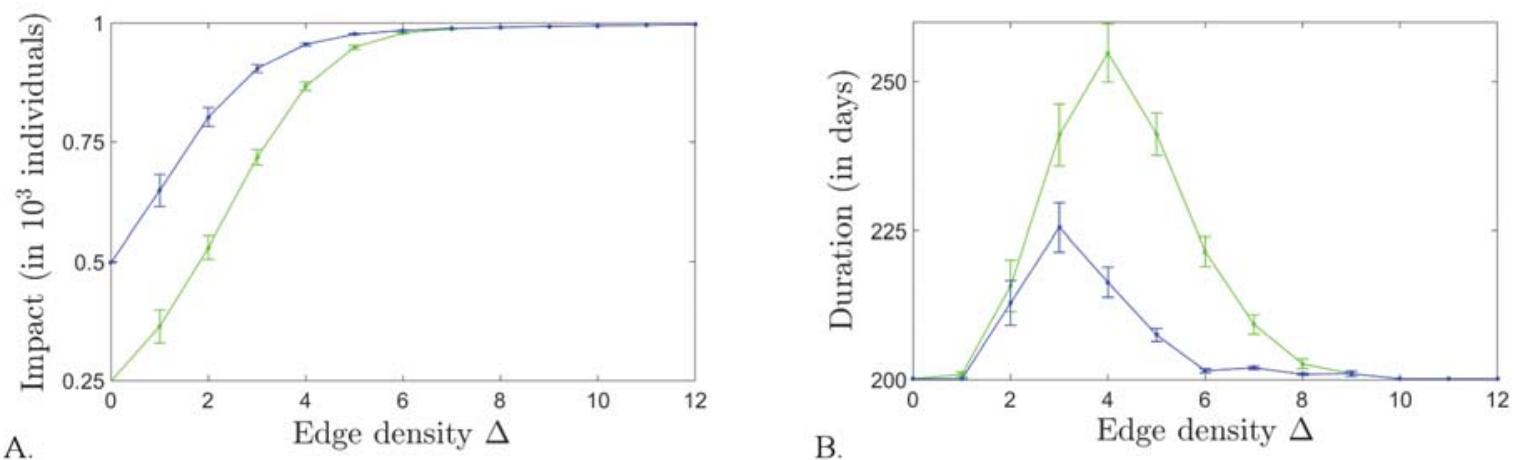

Figure 3. Dependence of the impact $R_{\infty}$ and of the outbreak duration $L$ on edge density $\Delta$. For a network of $n=4$ populations each originally with $N_{0}=1000$ individuals, we illustrate $R_{\infty}$ and $L$ as the edge density is varied for $\Delta=0$ edges to $\Delta=12$ edges. For each density value, the average impact and duration were computed over all possible $C_{n(n-1)}^{\Delta}$ adjacency configurations with the respective density. We considered the case of one focal point consisting of two latent cases, both in the same node (green curve), and the case of two focal points, each consisting of one latent case, starting simultaneously at two distinct nodes (blue curve).



A



B

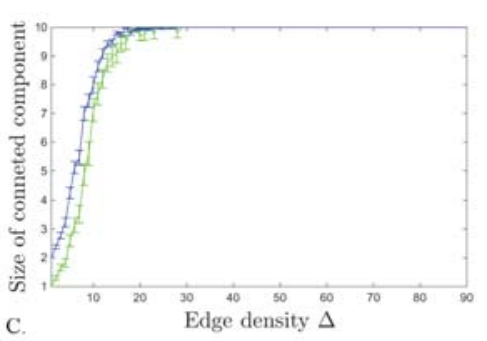

Figure 4. Dependence of the impact $R_{\infty}$ and of the outbreak duration $L$ on edge density $\Delta$. For a network of $n=10$ populations each originally with $N_{0}=1000$ individuals, we illustrate $R_{\infty}$ (in panel A) and $L$ (in panel B) as the edge density is varied. Panel C, shows the average number of nodes connected (via oriented graph paths)to the initially infection focus or foci as a function of edge density. For each density value, the average impact, duration and size of the infected connected components were computed over a sample of 50 adjacency matrices chosen at random, with the respective density. We considered the case of one focal point consisting of two latent cases, both in the same node (green curve), and the case of two focal points, each consisting of one latent case, starting simultaneously at two distinct nodes (blue curve).

although lifting quarantines and increasing travel may shorten duration (as suggested by our model), this is not a feasible policy, since it would lead to an unacceptable increase in casualties. Some of these aspects will be further discussed in the next section.

It is also worth noticing that the impact has a slower pick-up in the case of one original focus, while the duration is comparable for one or multiple foci in the low connectivity range, then significantly longer for one focus in the intermediate connectivity range. In other words: for intermediate internode communication, the impact is milder if the original infection starts in one single focus, but this scenario loses its advantage in the context of duration.

More precisely: in this preliminary model, $R_{\infty}$ is relatively low in average only for values of the edge density up to $\sim 20 \%$ (as illustrated for $n=4$ in Figure 3 and for $n=10$ in Figure 4 ). After crossing a window of very high sensitivity (in our figures, density $15-20 \%$ ), $R_{\infty}$ increases to its asymptotic value $N_{0}=1000$ (the total number of individuals per node). This predicts a devastating effect, describing an outbreak that would affect virtually everyone 
within the linked communities, even at relatively low communication levels.

Of course, however feared and somber the perspective of a global pandemic, this is not the prediction we expect in reality, based on our existing experience with Ebola outbreaks; hence the model should be revised to reflect more realistic trends. One major flaw of this preliminary model is the assumption of instantaneous and continuous disease spread whenever communication means exist between two nodes. This is clearly not a realistic condition, greatly overestimating the contagion spread, which further reflects in the unrealistically large values of $R_{\infty}$. Below, we update the model to refine this condition.

Existence of an oriented edge from $k$ to $j$ should only signify that direct communication is possible between the two nodes (e.g., there is a road connecting two villages), but not necessarily that this connection is used continuously, or maintained at the same level at all times. We introduce a simple way of accounting for this variability, while keeping the model compartmental (i.e., counting overall transfer rates, rather than keeping track of dynamics of individuals). At each time step $t$, each existing connection from some $k$ to some $j$ is used with a fixed probability $r_{k j}$. For each node, the probability of inward or outward travel can be tuned according to the local or global situation of the network. For example, the flow can be temporarily diminished or cut completely if the node needs to be quarantined to prevent infection spread. For our first analysis, we take the rate travel $r$ for simplicity to be constant throughout the outbreak process, and identical for all outgoing edges. In the following section, we will allow $r$ to adapt, producing a variable distribution of values over all the network edges, changing according to the implementation measures typically taken to minimize the impact of the outbreak.
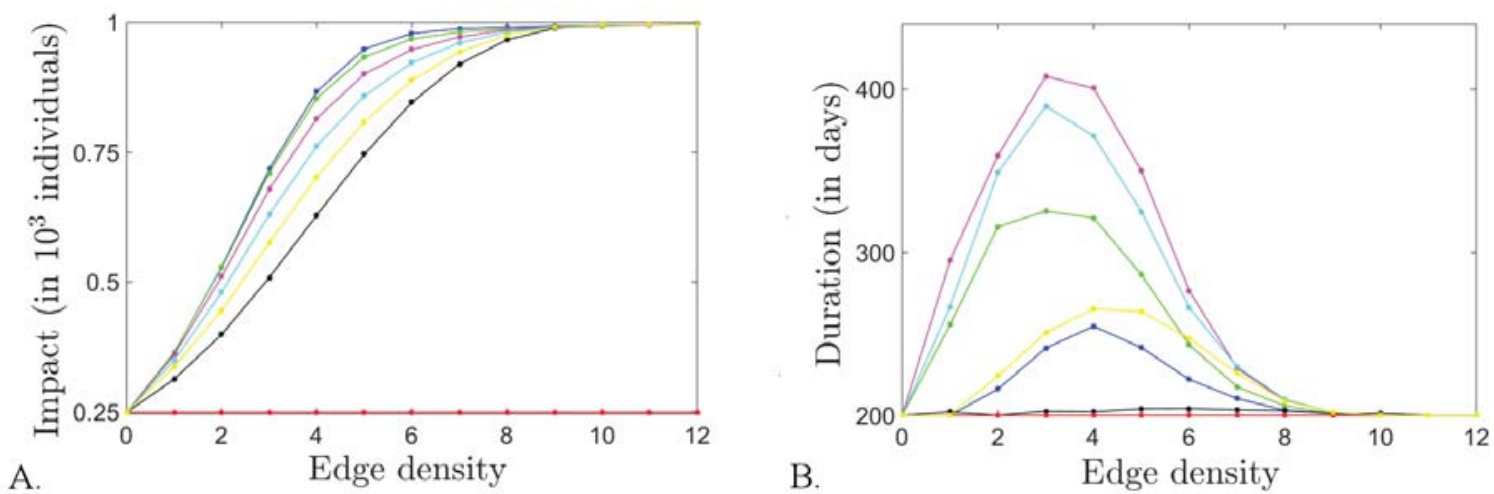

Figure 5. Dependence of outbreak aftermath on edge density $\Delta$ and travel likelihood $r$, for a network of $n=4$ communities. The impact $R_{\infty}$ (A) and outbreak duration (B) are computed, for each fixed edge density (number of 1s in the adjacency matrix) as averages over all the possible configurations of the adjacency matrix, with that fixed density. The different color plots correspond to different values of the probability to travel along an available edge: $r=1$ (blue), $r=0.5$ (green), $r=0.2$ (magenta), $r=0.1$ (cyan), $r=0.05$ (yellow), $r=0.01$ (black), $r=0$ (red).

Figure 5 illustrates in the case of probabilistic travel (described above), and for a single focal point of one individual, the dependence of impact and duration on both the density of edges, and on the probability $r$ to travel out of each node along the prescribed edges. Notice that impact and duration, while having the same qualitative dependence on density, exhibit subtle differences when changing the overall likelihood to travel $r$. For example, in the case of $n=4$ coupled nodes, the impact is $R_{\infty}=\frac{1}{4} N_{0}=250$ for $r=0$, since no travel implies that only the focal community will be affected (there is no dependence on edge density, since the edges are never used to transport the virus). Moving $r$ to any small positive value acts as a bifurcation, since the effects of the density $\Delta$ appear immediately, causing the impact to increase, more quickly and steeply for larger values of $r$. These being said, however, the dependence on travel likelihood in the $r>0$ range is not as dramatic in this model as one may 

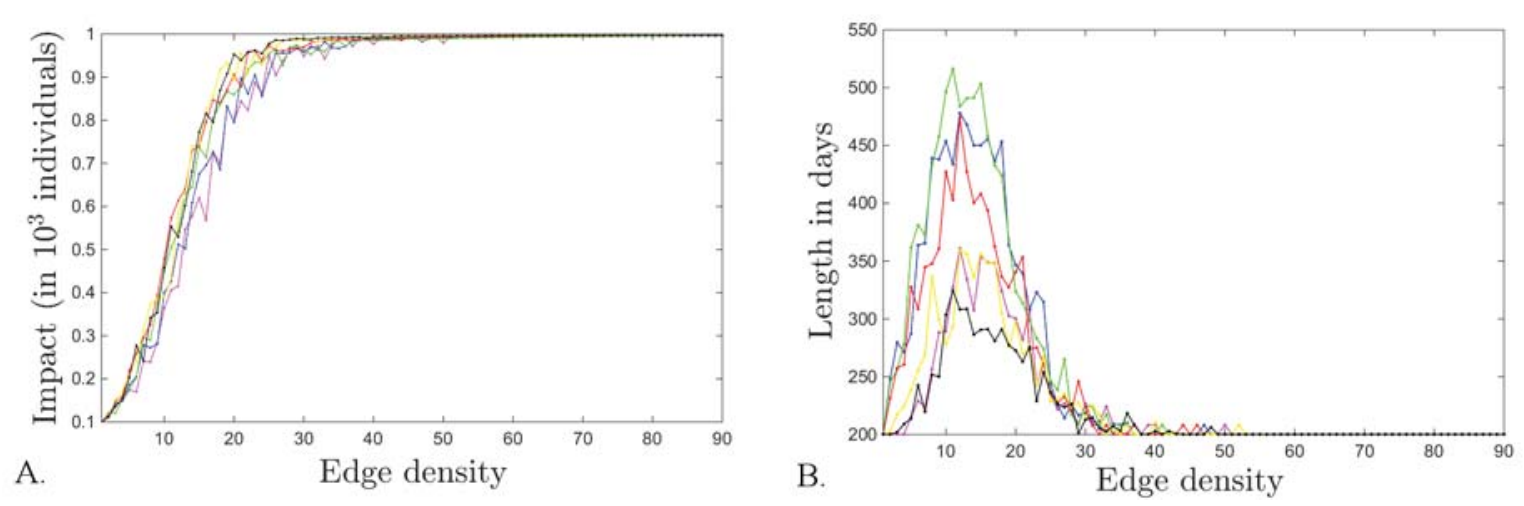

Figure 6. Dependence of outbreak aftermath on edge density $\Delta$ and travel likelihood $r$, for a network of $n=10$ communities. The impact $R_{\infty}$ (A) and of the outbreak duration (B) are computed, for each fixed edge density, as averages over a sample of $S=50$ adjacency configurations with that fixed density. The different color plots correspond to different values of the probability to travel along an available edge: $r=0.05$ (magenta), $r=0.1$ (blue), $r=0.2$ (green). $r=0.5$ (red), $r=0.8$ (yellow) and $r=1$ (black).

expect; it suggests that indiscriminately diminishing travel in the network can't single-handedly accomplish a major decrease in impact, unless the travel is altogether prohibited.

An even more surprising effect appears when studying the dependence on $r$ of the unimodal curve representing the outbreak duration (Figures $5 \mathrm{~b}$ and $6 \mathrm{~b}$ ). While the critical point does not vary much - remaining, for each curve, broadly in the same intermediate density range for each $n$ (a more precise localization could be obtained at the expense of lengthier computations), the behavior of the critical value differs both qualitatively and quantitatively between different $r$ values, first increasing with $r$, and then decreasing. This suggests that indiscriminately lowering $r$ may in fact be detrimental, by increasing the duration of the outbreak, without substantially lowering the impact (especially in the region of intermediate densities). Only a dramatic and implausible shift of $r$ to a value close to zero throughout the network would result in improving both impact and duration. These effects can be observed in both Figures 5 and 6, for $n=4$ and $n=10$, respectively.

Altogether it seems clear that, in order to control the outbreak in our model, one cannot just treat the system as a whole, but instead has to target more specific network sites, based on (1) the network architecture and (2) the outbreak's current state throughout the network, starting from the source of contagion.

\section{Modular adaptable network and effects of quarantine}

In this section, we will simulate an outbreak in the more realistic context of two interacting subnetworks (or modules), and introduce more structured quarantine measures, with the aim of reducing both impact and duration. As a plausible, but simplified scenario, we consider the modules to be organized as bubs, in the sense that each has a central node connected bidirectionally with all other nodes in the respective subnetwork, also allowing a specific number of additional random oriented edges between the other nodes of the respective module (as in the example pictured in Figure 7). This aims to represent the interaction of two large structures (e.g., countries), each organized as a network of smaller communities (e.g., towns, or villages). Such a multi-modular graph contains local, intra-modular connections between nodes (e.g., roads between villages), and long-distance, inter-modular connections. In our case we considered, for simplicity, a single inter-modular, bidirectional edge, running between the two central hub nodes (which could be seen as the only significant communication means between the two countries, e.g. - airports). 


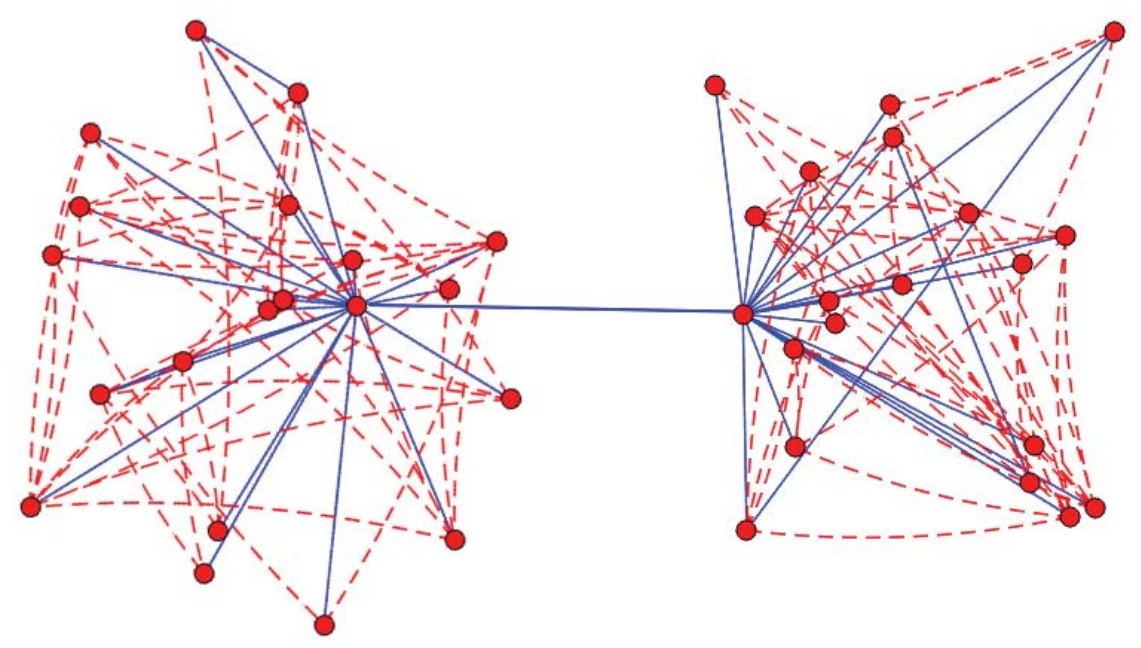

Figure 7. Model network architecture with two interconnected modules. Both modules (subgraphs), each with $n=20$ nodes and $\Delta=100$ oriented edges, are organized as hubs, each with one central node connected bidirectionally with all other nodes in the corresponding bub. The two hubs are connected by one single, bidirectional edge between their two central nodes. In this figure, bidirectional edges are represented in blue, and unidirectional edges are represented by dotted red curves, curved counter clock-wise. This type of architecture was used for the simulations, e.g., in Figure 8.

We discuss infection transmission and impact in this type of network, and the efficiency of introducing timely quarantines to control the outbreak. We distinguish between two types of quarantine, local (intra-modular) and global (inter-modular), as follows. If Ebola infection is detected for a period longer than $\theta$ days in a population/node (i.e., $I_{k}(t)>0$ for the specific node $P_{k}$ ), a local quarantine is introduced by cutting all in and out connections with the node $P_{k}$. If infection persists at any node within either hub for longer than $\tau$ days after the local quarantine (with $\tau>0$ ), then the two hubs are immediately disconnected (the connecting edge is cut off). We study how the timing of these isolation measures affects long term dynamics, towards finding a scheme that would minimize the inconvenience of lengthy quarantine, while still delivering efficient outbreak containment. While one naturally expects a prompter quarantine response to lead to a better outcome, our study looks in more detail at the extent to which the timing matters at both local and global levels.

We considered different architectures, edge densities and travel likelihoods $\left(r_{\text {int }}\right.$ along the active intra-modular edges and $r_{\text {ext }}$ along the inter-modular edge), at the start of the outbreak (time of the original infection in the network), and we measured in each case the dependence of the outbreak impact $R_{\infty}$ on the time delays $\theta$ and $\tau$ of the two quarantines. In Figure 8 we illustrate, for modules of size $n=10$, the dependence of $R_{\infty}$ on $\theta$ and $\tau$ (with each curve showing the dependence on $\tau$ for a different value of $\theta$, increasing from blue to green to red). The critical range (of maximum sensitivity) for $\theta$ is less than one day; the critical range for $\tau$ is a little longer. The top panels reproduce the outcome for a lower connectivity network (30 out of the maximum of 90 possible edges in each module); the bottom panels consider a higher connectivity network (50 edges). The left panels present results for lower travel likelihoods before the infection (intra-modular $r_{\text {int }}=0.4$ and $r_{\mathrm{ext}}=0.2$ along the inter-modular edge); the right panels show higher pre-infection travel likelihoods $\left(r_{\mathrm{int}}=r_{\mathrm{ext}}=0.5\right)$.

Generally, as one may expect, for any fixed local delay $\theta$, the impact increases with the inter-modular delay $\tau$ (the longer one waits to completely separate the two modules, the larger the infection-produced damage). Similarly, for each fixed $\tau$, the impact increases with the local delay $\theta$ (with the same wait time before separating the modules, a longer local delay increases the global average damage). However, these qualitative effects present wide quantitative 

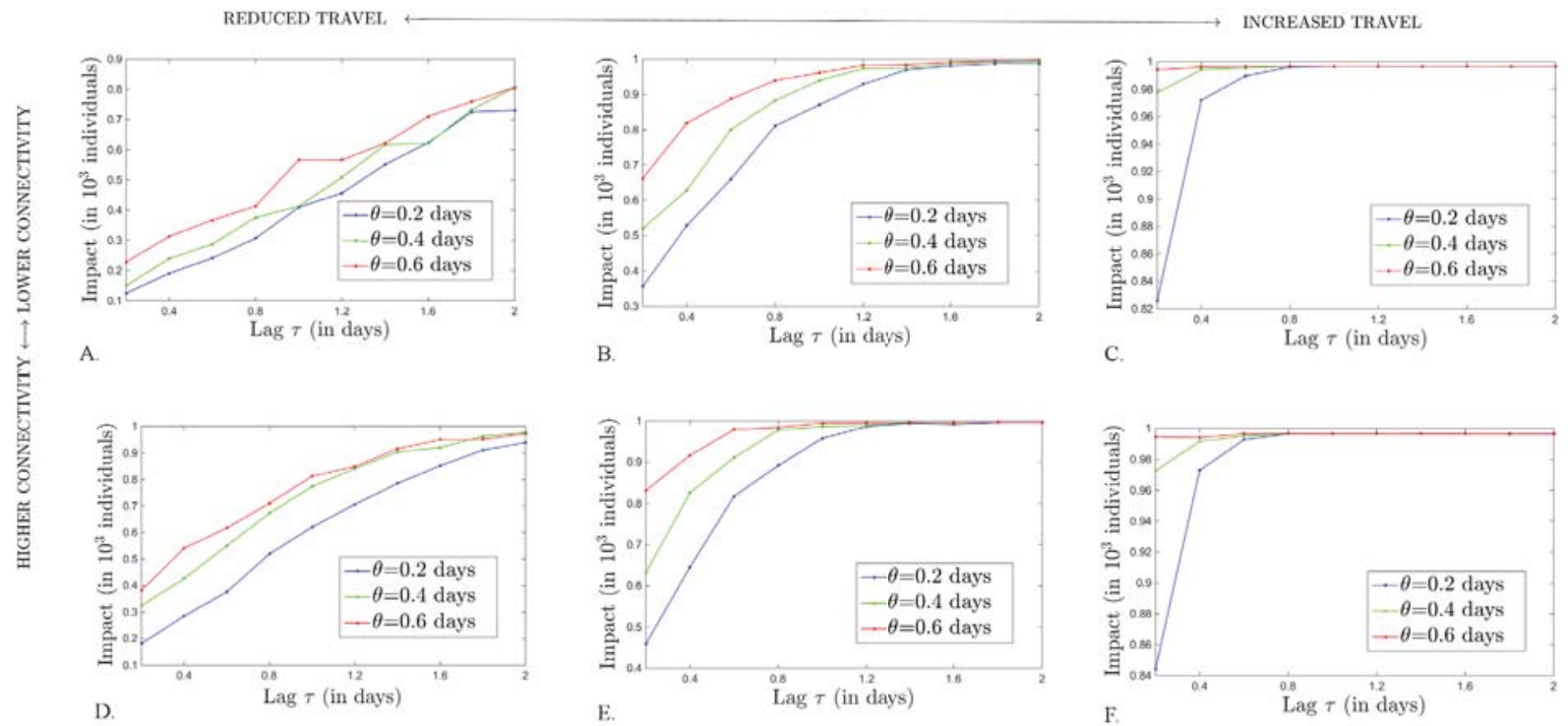

Figure 8. Dependence of impact $R_{\infty}$ on quarantine time lags $\theta$ and $\tau$ for a network composed of two bubs, each with $n=10$ nodes, connectivity density $\Delta=30$ and (top panels) and $\Delta=50$ (bottom panels) A. and $\mathbf{D}$. travel likelibood $r_{\text {int }}=0.2$ along intra-modular edges and $r_{\text {ext }}=0.1$ along the inter-modular edges; B. and E. travel likelibood $r_{\text {int }}=0.4$ along intra-modular edges and $r_{\text {ext }}=0.2$ along the inter-modular edges; C. and $\mathbf{F}$. travel likelihoods $r_{i n t}=r_{\text {ext }}=0.5$. Each curve represents the dependence of $R_{\infty}$ on the inter-modular lag $\tau$, for a fixed intra-modular lag $\theta$, as follows: $\theta=0.2$ days (in blue), $\theta=0.4$ days (in green) and $\theta=0.6$ days (in red).

variations with the structure and connectivity of the network.

One may note that, within the realistic connectivity range, there are no wide differences determined by the edge density per se (panels $\mathrm{A}$ and $\mathrm{D}$, as well as $\mathrm{B}$ and $\mathrm{E}$ have only subtle quantitative differences, and panels $\mathrm{C}$ and $\mathrm{F}$ look almost identical); the more substantial differences are induced by people's likelihood to travel along the existing edges before the quarantines are imposed.

For very low original travel rates along edges (left panels), local and global quarantines both drastically decrease impact in an almost linear fashion, with a slightly higher efficiency when shortening the global, rather than the local quarantine response.

For a network with medium travel along edges (center panels), the scenario is different: setting early quarantines has a very strong effect (the curves increase steeply for low values of $\tau$ ), effect which tapers off asymptotically as $\tau$ is relaxed. The impact also differs a lot with $\theta$, especially for values of $\tau$ less than one day (when the impact gets close to the common asymptotic value). In this case, it seems important to strive for a short local wait $\theta$. However, comparing the rates of change along each curve and across curves, it appears that shortening $\theta$ and $\tau$ has in this case comparable effects on the impact.

For highly traveled networks (right panels), a slightly large $\tau$ may lead to a catastrophic impact even for very low $\theta$ values. On the other hand, a large $\theta$ single-handedly leads to a dramatic impact, even with very short $\tau$. In such networks, only a combination of both small $\theta$ and small $\tau$ can substantially reduce the impact. An efficient control of the impact requires very quick local intervention, followed tightly, possibly even before observing success or failure of local measures, by global separation. 


\section{DISCUSSION}

In this paper, we investigated how the effects of an Ebola outbreak depend on the mobility of individuals in a network of connected populations exposed to the virus. This falls in the realm of a widely studied theoretical problem: that of dependence of coupled nonlinear dynamics on the architecture of the coupling. For our illustration, we worked with system parameters measured in a historic Ebola outbreak, but the same compartmental construction, the same concepts and methods can be used to study other viral transmissions, or any information diffusion process in a network of coupled nodes.

Aside from common sense conclusions on the necessity of prompt quarantines at early signs of a viral outbreak, our study brings interesting quantitative highlights, with potential applications when customizing and tuning quarantine placement and timing. There are a few particular observations that may be of general value when assessing the importance of quarantine and other measures to control an outbreak. First, we saw that conditions which minimize the outbreak impact may not be ideal for other aspects (e.g., duration), as important for the affected population. Second, a prompt local quarantine is generally helpful, but cannot efficiently lower the impact in and of itself, if not paired with a prompt global separation. A delay in global separation may make the efficiency of local quarantine irrelevant. Third, fast global separation is optimal if paired with a prompt local quarantine, and in some cases this can efficiently and substantially lower the impact.

This suggests that a helpful approach to infection control in a hypothetical outbreak would require authorities to assess the type of network and travel flow that is at risk, and tune the quarantine timing to occur within the respective optimal ranges. For example, in the case of a highly traveled modular network, the global separation may have to be imposed faster than common sense suggests, even if this may involve additional resources in putting in motion the slow global machinery.

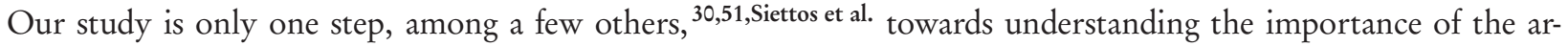
chitecture and hardwiring in a network exposed to an epidemic outbreak. It has clear limitations, but also a wide potential for extensions (to other disease dynamics, other population networks or even more general dynamic networks), and for more elaborate analyses of the underlying mathematical phenomena. Some of these aspects are discussed below.

One limitation introduced conceptually in the model relates to the strict assumptions made on Ebola spread and immunity. For example, we worked from the start under the hypothesis that the individuals who had contracted Ebola once, cannot have the disease again. This was based on a corresponding assumption in our original reference paper, ${ }^{4}$ which in turn was supported by the lack of evidence of any individuals with more than one Ebola infection within their life span. This idea is currently considered controversial in the disease dynamics literature, especially with the known variety of Ebola virus strains which may make a prior infection with one strain irrelevant immunologically to a new infection with a different strain. Immunity built-up aspects, such as duration and effectiveness to multiple viral strains, have been also explored mathematically. ${ }^{39}$

Another simplifying assumption we made was that infected people can no longer spread the virus after the infection clears, whether this occurs through recovery or death of the individual. In reality, this may not be accurate. While some studies show that the risk of transmission from bodily fluids of convalescent patients is low (when infection control guidelines for the viral hemorrhagic fevers are followed ${ }^{8}$ ), other studies have shown that the Ebola virus can be spread through the sperm of recovered individuals for up to six months after infection. ${ }^{14}$ It is also well known at this point that dead bodies can remain contagious for up to 60 days, ${ }^{2}$ with the potential of infection spread though contact with the dead body (e.g., during ritual funerals ${ }^{11}$ ). Further iterations of the model may consider introducing these effects into the coupled equations.

Finally, one important aspect to explore in future studies is the extent to which details of the network configuration 
can flip the optimal quarantine circumstances. The current paper shows that the quarantine measures required for maximal control may differ with measures such as edge density, or travel likelihood. Other studies have explored the impact of the small-worldness, or assortativity of the network on the overall dynamics. ${ }^{30}$ There is, however, the likely possibility that there is no canonically optimal response based only on global network measures, and that an adequate quarantine systems has to be customized in response to the local details of the network architecture. Returning to out original analogy between viral diffusion and brain dynamics - in the same fashion in which clinical neuroscience is evolving towards "brain profiling," and personalized clinical assessments, in the same way the response to a global Ebola outbreak may have to consider a "population network profiling" in order to deliver optimal results.

Our small values for optimal quarantine time lags suggest that full preparedness for a global outbreak may involve having a pre-established plan of action, to avoid fatal computation and decision-related delays during the spreading of the outbreak. This would require constantly updated knowledge of local and global travel patterns, a dynamic "global connectivity map" that could be implemented directly, when necessary, into simulations, and produce immediate predictions and provide efficient choices for quarantines. The cost of maintaining online global information may be a well placed investment in view of a potential pandemic.

\section{REFERENCES}

1. Adegun, O. (2014) The effects of Ebola virus on the economy of West Africa through the trade channel. IOSR Journal of Humanities and Social Science (IOSR-JHSS)(19) 10, 48-56.

2. Ahmad, K. (2014) Ebola virus infection: An overview. Journal of Islamabad Medical E Dental College (JIMDC) $3(2), 76-78$.

3. Anderson, R., May, R., and Anderson, B. (1992) Infectious diseases of humans: dynamics and control 28. Wiley Online Library.

4. Astacio, J., Briere, D., Guilléon, M., Martinez, J., Rodriguez, F., and Valenzuela-Campos, N. (1996) Mathematical models to study the outbreaks of Ebola. Biometrics Unit Technical Report, 1-18.

5. Bailey, N. (1975) The mathematical theory of infectious diseases and its applications. Charles Griffin \& Company Ltd, 5a Crendon Street, High Wycombe, Bucks HP13 6LE.

6. Barthélemy, M., Barrat, A., Pastor-Satorras, R., and Vespignani, A. (2004) Velocity and hierarchical spread of epidemic outbreaks in scale-free networks. Physical Review Letters 92(17), 178701.

7. Bassett, D. and Bullmore, E. (2006) Small-world brain networks. The Neuroscientist 12(6), 512-523.

8. Bausch, D., Towner, J., Dowell, S., Kaducu, F., Lukwiya, M., Sanchez, A., Nichol, S., Ksiazek, T., and Rollin, P. (2007) Assessment of the risk of Ebola virus transmission from bodily fluids and fomites. Journal of Infectious Diseases 196(Supplement 2), S142-S147.

9. Briand, S., Bertherat, E., Cox, P., Formenty, P., Kieny, M.-P., Myhre, J., Roth, C., Shindo, N., and Dye, C. (2014) The international Ebola emergency. New England Journal of Medicine 371(13), 1180-1183.

10. Bullmore, E. and Sporns, O. (2009) Complex brain networks: graph theoretical analysis of structural and functional systems. Nature Reviews Neuroscience 10(3),186-198.

11. Chippaux, J.-P. (2014) Outbreaks of Ebola virus disease in Africa: the beginnings of a tragic saga. J Venom Anim Toxins Incl Trop Dis 20(1), 44.

12. Chowell, G., Hengartner, N., Castillo-Chavez, C., Fenimore, P., and Hyman, J. (2004) The basic reproductive number of Ebola and the effects of public health measures: the cases of Congo and Uganda. Journal of Theoretical Biology 229(1), 119-126.

13. Danon, L., Ford, A., House, T., Jewell, C., Keeling, M., Roberts, G., Ross, J., and Vernon, M. (2011) Networks and the epidemiology of infectious disease. Interdisciplinary Perspectives on Infectious Diseases, 284909.

14. Deen, G., Knust, B., Broutet, N., Sesay, F., Formenty, P., Ross, C., Thorson, A., Massaquoi, T., Marrinan, J., Ervin, E., et al. (2015) Ebola RNA persistence in semen of Ebola virus disease survivors-a preliminary report. 
New England Journal of Medicine.

15. Enns, E., Mounzer, J., and Brandeau, M. (2012) Optimal link removal for epidemic mitigation: A two-way partitioning approach. Mathematical Biosciences 235(2),138-147.

16. Erickson, B., Widener, A., et al. (2014) Preparedness for Ebola questioned. Chemical E Engineering Nerws 92(42), 7-7.

17. Eubank, S., Guclu, H., Kumar, V., Marathe, M., Srinivasan, A., Toroczkai, Z., and Wang, N. (2004) Modelling disease outbreaks in realistic urban social networks. Nature 429(6988),180-184.

18. Feldmann, H. (2014) Ebola-a growing threat? New England Journal of Medicine 371(15), 1375-1378.

19. Frieden, T., Damon, I., Bell, B., Kenyon, T., and Nichol, S. (2014) Ebola 2014 - new challenges, new global response and responsibility. New England Journal of Medicine 371(13), 1177-1180.

20. Gostin, L., Lucey, D., and Phelan, A. (2014) The Ebola epidemic: a global health emergency. JAMA 312(11), 1095-1096.

21. Gross, T., D’Lima, C., and Blasius, B. (2006) Epidemic dynamics on an adaptive network. Physical Review Letters 96(20), 208701.

22. Haas, C. (2014) On the quarantine period for Ebola virus. PLoS Currents 6.

23. Heesterbeek, J. (2000) Mathematical epidemiology of infectious diseases: model building, analysis and interpretation 5. John Wiley \& Sons.

24. Hewlett, B. S. and Hewlett, B. L. (2007) Ebola, culture and politics: the anthropology of an emerging disease. Cengage Learning.

25. Hu, Z., Bi, P., Ma, W., Ruan, S., et al. (2011) Bifurcations of an SIRS epidemic model with nonlinear incidence rate. Discrete and Continuous Dynamical Systems B 15(1), 93-112.

26. Keeling, M. and Eames, K. (2005) Networks and epidemic models. Journal of the Royal Society Interface 2(4), 295-307.

27. Kenah, E. and Miller, J. (2011) Epidemic percolation networks, epidemic outcomes, and interventions. Interdisciplinary Perspectives on Infectious Diseases [P], 1-13.

28. Kenah, E. and Robins, J. (2007) Network-based analysis of stochastic SIR epidemic models with random and proportionate mixing. Journal of Theoretical Biology 249(4), 706-722.

29. Kiskowski, M. (2013) A three-scale network model for the early growth dynamics of 2014 West Africa Ebola epidemic. PLoS currents 6.

30. Kopman, S., Akbas, M., and Turgut, D. (2012) Epidemicsim: Epidemic simulation system with realistic mobility. In Local Computer Networks Workshops (LCN Workshops), IEEE 37th Conference on, 659-665.

31. Labouba, I. and Leroy, E. (2015) Ebola outbreaks in 2014. Journal of Clinical Virology 64, 109-110.

32. Legrand, J., Grais, R., Boelle, P.-Y., Valleron, A.-J., and Flahault, A. (2007) Understanding the dynamics of Ebola epidemics. Epidemiology and Infection 135(4), 610-621.

33. Lekone, P. and Finkenstädt, B. (2006) Statistical inference in a stochastic epidemic SEIR model with control intervention: Ebola as a case study. Biometrics 62(4), 1170-1177.

34. Lewnard, J., Mbah, M., Alfaro-Murillo, J., Altice, F., Bawo, L., Nyenswah, T., and Galvani, A. (2014) Dynamics and control of Ebola virus transmission in Montserrado, Liberia: a mathematical modelling analysis. The Lancet Infectious Diseases 14(12), 1189-1195.

35. Marceau, V., Noël, P.-A., Hébert-Dufresne, L., Allard, A., and Dubé, L. (2011) Modeling the dynamical interaction between epidemics on overlay networks. Physical Review E 84(2), 026105.

36. Moreno, Y., Pastor-Satorras, R., and Vespignani, A. (2002) Epidemic outbreaks in complex heterogeneous networks. The European Physical Journal B-Condensed Matter and Complex Systems 26(4), 521-529.

37. Newman, M. (2003) The structure and function of complex networks. SIAM Revierw 45(2),167-256.

38. Pandey, A., Atkins, K., Medlock, J., Wenzel, N., Townsend, J., Childs, J., Nyenswah, T., Ndeffo-Mbah, M., and Galvani, A. (2014) Strategies for containing Ebola in West Africa. Science 346(6212), 991-995.

39. Piazza, N. and Wang, H. (2013) Bifurcation and sensitivity analysis of immunity duration in an epidemic 
model. Int. J. Numer. Analy. And Modeling Series B Computing and Information 4, 179-202.

40. Read, J., Eames, K., and Edmunds, W. (2008) Dynamic social networks and the implications for the spread of infectious disease. Journal of The Royal Society Interface 5(26),1001-1007.

41. Richards, P., Amara, J., Ferme, M., Kamara, P., Mokuwa, E., Sheriff, A., Suluku, R., and Voors, M. (2015) Social pathways for Ebola virus disease in rural Sierra Leone, and some implications for containment. PLoS Negl Trop Dis 9(4), e0003567.

42. Rivers, C., Lofgren, E., Marathe, M., Eubank, S., and Lewis, B. (2014) Modeling the impact of interventions on an epidemic of Ebola in Sierra Leone and Liberia. PLoS currents 6.

43. Rădulescu, A. and Verduzco-Flores, S. (2015) Nonlinear network dynamics under perturbations of the underlying graph. Chaos: An Interdisciplinary Journal of Nonlinear Science 25(1), 013116.

44. Safi, M., Imran, M., and Gumel, A. (2012) Threshold dynamics of a non-autonomous SEIRS model with quarantine and isolation. Theory in Biosciences 131(1),19-30.

45. Satsuma, J., Willox, R., Ramani, A., Grammaticos, B., and Carstea, A. (2004) Extending the SIR epidemic model. Physica A: Statistical Mechanics and its Applications 336(3), 369-375.

46. Schieffelin, J., Shaffer, J., Goba, A., Gbakie, M., Gire, S., Colubri, A., Sealfon, R., Kanneh, L., Moigboi, A., Momoh, M., et al. (2014) Clinical illness and outcomes in patients with Ebola in Sierra Leone. New England Journal of Medicine 371(22), 2092-2100.

Siettos et al.. Siettos, C., Anastassopoulou, C., Russo, L., Grigoras, C., and Mylonakis, E. (2015) Modeling the 2014 Ebola virus epidemic-agent-based simulations, temporal analysis and future predictions for Liberia and Sierra Leone. PLoS Currents 7.

48. Smieszek, T., Fiebig, L., and Scholz, R. (2009) Models of epidemics: when contact repetition and clustering should be included. Theoretical Biology and Medical Modelling 6(1), 11.

49. Stehlé, J., Voirin, N., Barrat, A., Cattuto, C., Colizza, V., Isella, L., Régis, C., Pinton, J.-F., Khanafer, N., Van den Broeck, W., et al. (2011) Simulation of an SEIR infectious disease model on the dynamic contact network of conference attendees. BMC Medicine 9(1), 87.

50. Ströher, U. and Feldmann, H. (2006) Progress towards the treatment of Ebola haemorrhagic fever. Expert Opinion on Investigational Drugs 15(12), 1523-1535.

51. Valdano, E., Ferreri, L., Poletto, C., and Colizza, V. (2015) Analytical computation of the epidemic threshold on temporal networks. Physical Review X 5(2), 021005.

52. van den Heuvel, M. and Sporns, O. (2011) Rich-club organization of the human connectome. The Journal of Neuroscience 31(44), 15775-15786.

53. Zelazo, D. and Mesbahi, M. (2008) On the observability properties of homogeneous and heterogeneous networked dynamic systems. In 47th IEEE Conference on Decision and Control, 2997-3002.

\section{ABOUT THE STUDENT AUTHOR}

Joanna Herron is currently a senior at SUNY New Paltz, pursuing a Bachelor of Science in mathematics and psychology. She has had the opportunity to help conduct a variety of research in areas from game studies to computational neuroscience during her undergraduate career, and hopes to work in a behavioral laboratory after graduation.

\section{PRESS SUMMARY}

We define the impact and the duration of a viral outbreak in a network model of an infected population. We study the dependence of these measures on local and global network connectivity, and on the travel likelihood within the network. We perform simulations of the outbreak course, first in a network with fixed travel rates, then in an adaptable network with two connected hubs. In particular, we study the effects that quarantine can have on the impact and duration, for different possibilities of network hardwiring. We find that quarantine options need to be specific in order to alleviate outbreak effects. 